\title{
Correlation Between Divided Doses of Chronic Antihypertensive Drugs and Pregnancy Outcomes
}

\author{
Safura Hatamipour Dehno ${ }^{1}$, Simin Taghavi $^{{ }^{*}}$, Nayyereh Ayati ${ }^{2}$
}

\begin{abstract}
Objectives: Hypertension, as a common problem during pregnancy, is a major cause of maternal and fetal morbidity and mortality. Anti-hypertensive drugs are used to prolong the pregnancy or modify perinatal outcomes in pregnant women with hypertensive disorders. Severe monitoring of blood pressure is subsequently essential in these mothers. The aim of this study was to evaluate the correlation between divided doses of chronic hypertensive drugs and the end of pregnancy in mothers with gestational hypertension. Materials and Methods: In this prospective research, 99 pregnant women with chronic hypertension, who were treated with antihypertensive medicines, were studied during pregnancy. During routine follow-up of these mothers, the number of the drug and the divided doses were recorded. The incidence of maternal and fetal outcomes were evaluated according to the number of less or more than 5 divided doses.

Results: Maternal and fetal-baby complications were observed in 5.50 and 5.53\% cases, respectively. The incidence of maternal and fetal complications were significantly enhanced by increasing the number of up-taken anti-hypertensive drugs. The maternal and fetal-baby complications were higher in mothers who received more than five divided doses compared to those who received less than five doses.

Conclusion: Based on our results, chronic hypertension was associated with maternal and fetal-baby complications in half of the cases. It is clarified that precise blood pressure monitoring and regularly taking of anti-hypertensive medicine may decrease the hypertension side effects. The awareness about this field in the pre-pregnancy consulting is considered as the patients' rights and should be respected.
\end{abstract}

Keywords: Hypertension, Pregnancy, Pregnancy outcome

\section{Introduction}

Hypertension is a common disorder during pregnancy (1) and is considered a major cause of maternal and fetal-neonatal mortality and morbidity worldwide (1-3). Several complications are associated with hypertension including fetal-neonatal and maternal mortality (3-5), preterm delivery (6), low weight for gestational age (6$8)$, intrauterine growth restriction (IUGR) (9), placental abruption (10), increased risk of Cesarean section delivery, heart failure, acute renal failure, and HELLP syndrome. People with high blood pressure during pregnancy are also at high risk of cardiovascular disease, diabetes and obesity (8). In the United States, $17.6 \%$ of maternal deaths are caused by hypertension during pregnancy (11).

Maternal age, maternal weight, specific ethnicities, multiple pregnancies and other underlying diseases such as chronic hypertension, diabetes mellitus (DM) and systemic lupus erythematosus are among its risk factors (12).

Diagnosis of chronic hypertension in pregnancy should be confirmed. After diagnosis, the objective is to reduce the negative maternal and fetal-neonatal outcomes.
Treatments are aimed at preventing the exacerbation of hypertension and postponing or treating the pregnancyinduced hypertension that can occur in the near future. These objectives can be somewhat achieved through administration of medications and frequent evaluation of the mother's body systems.

The use of antihypertensive drugs in an attempt to prolong the pregnancy or improve the perinatal outcomes in pregnant women with hypertensive disorders of different intensity has been of special interest to physicians and researchers.

The medications used to control blood pressure in pregnancy include diuretics, beta-adrenergic receptor blocking drugs (e.g., methyldopa and labetalol), vascular dilator (e.g., hydralazine), and calcium channel blockers (e.g., nifedipine, verapamil) (13-17).

It is suggested that a more rigorous control of blood pressure reduces the pregnancy complications (18). No study has been conducted on the exact role of number of antihypertensive medications doses on maternal and fetal-neonatal outcomes. Therefore, the aim of this study was to evaluate the correlation between number of divided 
doses of chronic antihypertensive drugs and pregnancy outcome in women with chronic pregnancy hypertension.

\section{Materials and Methods}

A total of 99 pregnant women with chronic hypertension who were treated with antihypertensive medicines in pregnancy, such as methyldopa, hydralazine, nifedipine, labetalol, diuretics and visited Alzahra educational health center during March 2015-Februry 2016 were enrolled in the study after obtaining consent.. Blood pressures of all mothers were measured at each visit using a standard sphygmomanometer, and dose and type of medication were increased or decreased, if needed (Table 1: required drug type and dose).

Dose adjustments aimed at maintaining blood pressure at $90-100 / 150$.

After 1 or 2 weeks, and once the dose necessary to control blood pressure were fixed, patients were divided into one of following groups based on the number of divided doses:

- Group 1: Requiring 2 divided doses

- Group 2: Requiring 3 divided doses

- Group 3: Requiring 4 divided doses

- Group 4: Requiring 5 divided doses

- Group 6: Requiring 6 divided doses

- Group 7: Requiring 7 divided doses

During treatment, if an increase or decrease in the dose of medication was needed, the patient would not be transferred to another group. In this case, the increase or decrease in dose was considered a pregnancy outcome.

Maternal outcomes including pre-eclampsia, HELLP syndrome, cerebral stroke, acute kidney injury, heart failure, hypertensive cardiomyopathy, myocardial infarction, placental abruption, and fetal-neonatal outcomes including fetal death, growth restriction, preterm delivery, fetal death, and neonatal morbidity were evaluated at each visit. For each patient, prescribed medications, dosage, number of required divided doses, and type of negative maternal and fetal-neonatal outcomes were recorded. Maternal and neonatal outcomes were defined in six categories. Ultimately, patients receiving less than 5 doses and patients receiving more than 5 doses were compared.

Table 1. Incidence of Fetal-Neonatal Complication in Subjects Receiving Less or More Than 5 Divided Doses of Antihypertensive Drug

\begin{tabular}{lccc}
\hline & $\begin{array}{c}\mathbf{5} \text { and More } \\
\text { Doses }\end{array}$ & $\begin{array}{c}\text { Less Than } \mathbf{5} \\
\text { Doses }\end{array}$ & P Value \\
\hline $\begin{array}{l}\text { Fetal overall } \\
\text { complications }\end{array}$ & $22(81.5 \%)$ & $31(43.1 \%)$ & 0.001 \\
Fetal death & $4(14.8 \%)$ & $3(4.2 \%)$ & 0.06 \\
IUGR & $3(11.1 \%)$ & $4(5.6 \%)$ & 0.38 \\
Preterm birth & $13(48.1 \%)$ & $33(45.8 \%)$ & 0.9 \\
Baby death & $2(4.7 \%)$ & $2(2.8 \%)$ & 0.29 \\
Baby morbidity & $3(11.1 \%)$ & $4(5.6 \%)$ & 0.33 \\
\hline
\end{tabular}

Statistical Analysis

All data were analyzed using SPSS 17. Descriptive statistics (frequency, percentage, mean \pm standard deviation) were used for statistical analysis. All results with a $P$ value $<0.05$ were considered statistically significant.

\section{Results}

In this study, 99 pregnant women with chronic pregnancy hypertension were examined. The mean age of the mothers was $32.85 \pm 6.31$ years at a median of 34 years. The youngest and oldest patients were 18 and 46 years old. Mean gravidity of patients was $2.72 \pm 1.55$ at a median of 3. Minimum and maximum gravidity were 1 and 7 , respectively. Mean para of mothers was $1.16 \pm 1.13$ at a median of 1 . Minimum and maximum para were 0 and 5 , respectively. The mean number of miscarriages in mothers was $0.55 \pm 0.88$ at a median of 0 . The lowest and highest number of miscarriages was 0 and 4, respectively. The average number of live births was $1.03 \pm 1.08$ at a median of 1 . Minimum and maximum number of live births was 0 and 5, respectively. Of 99 mothers, 98 (99\%) were housewives and one (1\%) was employed. Seventy mothers $(70.7 \%)$ resided in urban areas, while 29 (29.3\%) resided in rural areas.

With respect to the history of the disease and complications in previous pregnancies, no one had a history of placental abruption, 8 subjects had a history of pre-eclampsia (8.1\%), 3 had a history of superimposed pre-eclampsia (3\%), 11 had a history of DM (11.1\%), 9 had a history of IUGR $(9.1 \%)$, and one had a history of chronic kidney disease (1\%). The mean number of drugs used by the patients was $1.30 \pm 0.59$ at a median of 1 . The lowest and highest number of drugs received was 1 and 3 , respectively. The mean received divided doses were $3.26 \pm 1.78$ at a median of 2 doses. The lowest and highest doses were 1 and 9 , respectively.

Figure 1 shows the frequency of divided doses of antihypertensive drugs received by the pregnant mothers. As can be seen, the majority of patients were receiving 2 or less doses.

\section{Maternal Complications}

Maternal complications were observed in 50 subjects $(P<0.001)$. These complications include placental abruption in 5 subjects (5.1\%), pre-eclampsia in 44 subjects $(44.4 \%)$, superimposed pre-eclampsia in 6 subjects (6.1\%), HELLP in 10 subjects (10.1\%), acute renal failure in one subject (1\%), and heart failure in two subjects $(2 \%)$. In some cases, more than one complication was present. No maternal mortality was observed.

Fetal-Neonatal Complications

Fetal-neonatal complications were observed in 53 ( $P$ $<0.001)$ of the infants. These complications included fetal death in 7 cases $(7.1 \%)$, infant death in 4 cases (4\%), IUGR in 7 cases (7.1\%), preterm delivery in 46 cases (46.5\%) and 
Table 2. The Incidence of Maternal and Fetal-Neonatal Complications by Number of Drugs and Number of Divided Doses

\begin{tabular}{|c|c|c|c|c|}
\hline \multicolumn{5}{|c|}{ Maternal Complications } \\
\hline & & With Maternal Complications & Without Maternal Complications & P Value \\
\hline \multirow{3}{*}{ Number of drugs } & 1 & $32(42.1 \%)$ & $44(57.9 \%)$ & \\
\hline & 2 & $12(75 \%)$ & $4(25 \%)$ & 0.009 \\
\hline & 3 & $6(85.7 \%)$ & $1(14.3 \%)$ & \\
\hline The average of the up-taken drug & & $1.48 \pm 0.70$ & $1.12 \pm 0.38$ & 0.002 \\
\hline The average of the up-taken doses & & $4.00 \pm 1.81$ & $2.51 \pm 1.41$ & $<0.001$ \\
\hline 1 dose & & $1(7.7 \%)$ & $12(92.3 \%)$ & \\
\hline 2 dose & & $13(40.6 \%)$ & $19(59.4 \%)$ & \\
\hline 3 dose & & $10(62.5 \%)$ & $6(37.5 \%)$ & \\
\hline 4 dose & & $3(27.3 \%)$ & $8(72.7 \%)$ & \\
\hline 5 dose & & $14(87.5 \%)$ & $2(12.5 \%)$ & \\
\hline 6 dose & & $4(80 \%)$ & $1(20 \%)$ & \\
\hline 7 dose & & $4(80 \%)$ & $1(20 \%)$ & \\
\hline 9 dose & & $1(100 \%)$ & 0 & \\
\hline \multicolumn{5}{|c|}{ Fetal Complications } \\
\hline & & With Fetal Complications & Without Fetal Complications & P Value \\
\hline \multirow[t]{3}{*}{ Number of drugs } & 1 & $35(46.1 \%)$ & 41 (53.9\%) & \\
\hline & 2 & $12(75 \%)$ & $4(25 \%)$ & 0.02 \\
\hline & 3 & $6(85.7 \%)$ & $1(14.3 \%)$ & \\
\hline The average of the up-taken drug & & $1.45 \pm 0.69$ & $1.13 \pm 0.40$ & 0.007 \\
\hline The average of the up-taken doses & & $3.77 \pm 1.88$ & $2.67 \pm 1.47$ & 0.005 \\
\hline 1 dose & & $3(23.1 \%)$ & $10(76.9 \%)$ & \\
\hline 2 dose & & $15(46.9 \%)$ & $17(53.1 \%)$ & \\
\hline 3 dose & & $11(68.8 \%)$ & $5(31.3 \%)$ & \\
\hline 4 dose & & $2(18.2 \%)$ & $9(81.8 \%)$ & \\
\hline 5 dose & & $13(81.3 \%)$ & $3(18.8 \%)$ & \\
\hline 6 dose & & $4(80 \%)$ & $1(20 \%)$ & \\
\hline 7 dose & & $4(80 \%)$ & $1(20 \%)$ & \\
\hline 9 dose & & $1(100 \%)$ & 0 & \\
\hline
\end{tabular}

morbidity (mainly jaundice) in 7 cases $(7.1 \%)$.

Table 2 shows the incidence of maternal and neonatal complications by the number of drugs and the number of divided doses. The incidence of maternal and fetalneonatal complications was considerably increased by increase in the number of received antihypertensive drugs. The mean number of drugs and the number of doses received by mothers were considerably increased in mothers with maternal complications and those with infants with neonatal complications. Twenty-seven mothers $(27.3 \%)$ received more than 5 divided doses, and 72 mothers $(72.7 \%)$ received less than 5 divided doses of antihypertensive drugs during pregnancy.

Table 3 shows the incidence of maternal complications in subjects who received less than 5 divided doses and those who received more than 5 divided doses. As can be seen, the incidence of maternal complications was considerably higher in those who received 5 or more divided doses. Assessment of the individual complications showed that frequency of major complications was higher in subjects who received 5 or more divided doses; however, the difference was not statistically significant.

Table 1 shows the fetal-neonatal complications in subjects who received less than 5 divided doses and those who received 5 or more divided doses. The incidence of fetal-neonatal complications was considerably higher in mothers who received 5 or more divided doses. Assessment of the individual complications showed that incidence of complications was higher in these mothers compared to those who received less than 5 divided doses; however, the difference was not statistically significant.

\section{Discussion}

Pregnancy-induced hypertension is a major cause of morbidity and mortality in pregnant women, making its appropriate treatment necessary (19). Differentiation of chronic hypertension from its other manifestations or other comorbidities is very important because this disease can be considered dangerous in the maternity ward and must be treated immediately and then followed up until the end of pregnancy $(20,21)$. Chronic hypertension is observed in approximately $1 \%-5 \%$ of all pregnancies (22). El Guindy and Nabhan reported that pregnant women who had lower blood pressure control, compared to those whose blood pressure was strict controlled, had a higher hospitalization rates before labor and gave birth to infants with lower mean gestational age (18). Various drug therapies have been proposed in this regard. If blood pressure in not controlled by a single drug, the number of drugs and the number of doses must be increased, which 
Table 3. Incidence of Maternal Complication in Patients Receiving Less or More Than 5 Divided Doses of Antihypertensive Drug

\begin{tabular}{lccc}
\hline & $\begin{array}{c}\mathbf{5} \text { and More } \\
\text { Doses }\end{array}$ & $\begin{array}{c}\text { Less Than } \\
\mathbf{5} \text { Doses }\end{array}$ & P Value \\
\hline Maternal overall complications & $23(85.2 \%)$ & $27(37.5 \%)$ & $<0.001$ \\
Placental abruption & $3(11.1 \%)$ & $2(2.8 \%)$ & 0.12 \\
Pre-eclampsia & $14(51.9 \%)$ & $30(41.7 \%)$ & 0.37 \\
Pre-eclampsia superimposed & $1(3.7 \%)$ & $5(6.9 \%)$ & 0.54 \\
HELLP & $5(18.5 \%)$ & $5(6.9 \%)$ & 0.08 \\
Acute renal failure & $1(3.7 \%)$ & $0(0)$ & 0.27 \\
Heart failure & $2(7.4 \%)$ & $0(0)$ & 0.07 \\
\hline
\end{tabular}

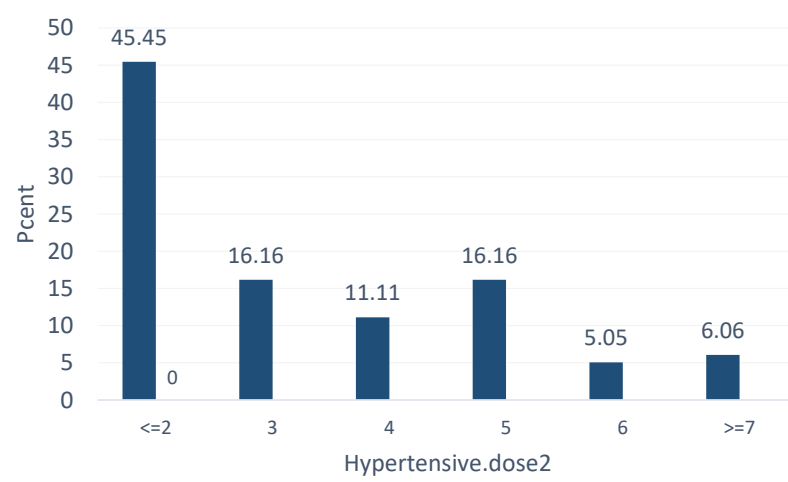

Figure 1. Frequency of Received Divided Doses of Antihypertensive Drug in Pregnant Women With Chronic Hypertension.

can be associated with adverse consequences.

The correlation between the numbers of divided doses of antihypertensive drugs in chronic hypertension and pregnancy was examined. It was observed that an increased number of drugs and number of doses received by patients increased the incidence of maternal and fetal complications. Antihypertensive drug treatment options in pregnancy have been limited to relatively safe drugs that have had a long clinical history with acceptable complications. Guidelines recommend administration of methyldopa and hydralazine as main drug treatments (23).

In this study, the most common antihypertensive drug was methyldopa, followed by hydralazine and betablockers. Other drugs included diltiazem, nifedipine, and labetalol. In this study, $76.8 \%$ of subjects used one drug, $16.2 \%$ used two drugs, and $7.1 \%$ used 3 drugs. The mean received divided dose was $3.26 \pm 1.78$ doses. In a study by Georges et al, $49.2 \%$ of subjects used only 1 antihypertensive drug, and $36.5 \%$ of subjects were treated with at least 2 drugs (24).

In the present study, maternal complications were observed in $50.5 \%$ of subjects, and included pre-eclampsia
(44.4\%), HELLP (10.1\%), superimposed pre-eclampsia (6.1\%), placental abruption (5.1\%), heart failure (2\%) and acute renal failure (1\%). Fetal-neonatal complications were observed in 53.5\% infants, and included preterm delivery (46.5\%), fetal death, IUGR and morbidity each in $7.1 \%$ of infants, and neonatal death (4\%). No case of maternal mortality was observed in this study.

According to a report by American College of Obstetrics and Gynecology (ACOG) in 2012, about 20\% of women with chronic hypertension in pregnancy developed superimposed pre-eclampsia (25). Georges et al observed eclampsia in $19.8 \%$ of mothers and no cases of maternal mortality. Intrauterine fetal death (IUFD) and premature infants were observed in $14.5 \%$ and $28.3 \%$ of cases, respectively (24).

In this present study, the relationship between the number of drugs and number of doses of anti-hypertensive drugs with fetal-neonatal and maternal complications was investigated, which has been mainly overlooked by previous studies. An increased number of antihypertensive drugs considerably increased the incidence of maternal and fetal complications. The incidence of maternal and fetal-neonatal complications was higher in mothers receiving 5 of more divided doses of antihypertensive drugs compared to those receiving less than 5 divided doses.

Unfortunately, the need for divided and multiple doses of the drugs can cause irregular use of medications. Pregnant women may not continuously use the drugs when the required number of doses of antihypertensive drug increases. Therefore, irregular use of drugs to control blood pressure can cause maternal and fetal complications.

\section{Conclusion}

Although pregnancy in women with chronic hypertension is associated with maternal and fetal-neonatal complication in half the cases, rigorous control of blood pressure and timely prescription of antihypertensive can obviously reduce such complications.

The risk of maternal and fetal-neonatal complications in pregnancy was considerably higher in women with chronic hypertension who received 5 or more than 5 divided doses of hypertensive drugs compared to those who received less than 5 doses. Awareness in this regard in counseling before pregnancy is a patient's right which must be respected.

\section{Conflict of Interests}

The authors declare no conflict of interests.

\section{Ethical issues}

The study was approved by the Committee on the Ethics of Tabriz University of Medical Sciences (NO. 5/4/11415). Informed consent was obtained from patients for using samples. 


\section{References}

1. James PR, Nelson-Piercy C. Management of hypertension before, during, and after pregnancy. Heart. 2004;90 (12):1499-1504. doi:10.1136/hrt.2004.035444.

2. Amira OC, Okubadejo NU. Frequency of complementary and alternative medicine utilization in hypertensive patients attending an urban tertiary care centre in Nigeria. BMC Complement Altern Med. 2007;7:30. doi:10.1186/14726882-7-30.

3. Roberts JM, Pearson G, Cutler J, Lindheimer M. Summary of the NHLBI Working Group on research on hypertension during pregnancy. Hypertension. 2003;41 (3):437-445. doi:10.1161/01.hyp.0000054981.03589.e9.

4. Chen XK, Wen SW, Smith G, Yang Q, Walker M. Pregnancyinduced hypertension is associated with lower infant mortality in preterm singletons. BJOG. 2006;113 (5):544551. doi:10.1111/j.1471-0528.2006.00898.x.

5. Zhang J, Cai WW, Lee DJ. Pregnancy-induced hypertension and early neonatal death: a case-control study. Am J Perinatol. 1993;10 (5):401-403. doi:10.1055/s-2007-994772.

6. Mugo M, Govindarajan G, Kurukulasuriya LR, Sowers JR, McFarlane SI. Hypertension in pregnancy. Curr Hypertens Rep. 2005;7 (5):348-354.

7. Madadzadeh M, Feizi Z, Hagikazemi ES, Haghani H. Mothers' hypertension and neonatal birth weight. Iranian J Neonatal. 2005;18 (41):17-25.

8. Miranda ML, Swamy GK, Edwards S, Maxson P, Gelfand A, James S. Disparities in maternal hypertension and pregnancy outcomes: evidence from North Carolina, 19942003. Public Health Rep. 2010;125 (4):579-587.

9. Chen XK, Wen SW, Smith GN, Yang Q, Walker MC. New-onset hypertension in late pregnancy and fetal growth: different associations between singletons and twins. Hypertens Pregnancy. 2007;26 (3):259-72. doi:10.1080/10641950701366825.

10. Huang Y. Incidence of pregnancy induced hypertension and the effects on mother and fetus in Shanghai during 1989-1998. Zhonghua Fu Chan Ke Za Zhi. 2001;36 (3):1379. [Chinese].

11. Hutcheon JA, Lisonkova S, Joseph KS. Epidemiology of pre-eclampsia and the other hypertensive disorders of pregnancy. Best Pract Res Clin Obstet Gynaecol. 2011;25 (4): 391-403. doi:10.1016/j.bpobgyn.2011.01.006.

12. Wright D, Akolekar R, Syngelaki A, Poon LC, Nicolaides KH. A competing risks model in early screening for preeclampsia. Fetal Diagn Ther. 2012;32 (3):171-178. doi:10.1159/000338470.

13. Brown CM, Garovic VD. Drug treatment of hypertension in pregnancy. Drugs. 2014;74 (3):283-96. doi:10.1007/ s40265-014-0187-7.

14. Su CY, Lin HC, Cheng HC, Yen AM, Chen YH, Kao S. Pregnancy outcomes of anti-hypertensives for women with chronic hypertension: a population-based study. PLoS ONE 2013;8 (2):e53844. doi:10.1371/journal.pone.0053844.

15. Podymow T, August P. Antihypertensive drugs in pregnancy. Semin Nephrol. 2011;31 (1):70-85. doi:10.1016/j. semnephrol.2010.10.007.

16. Easterling TR. Pharmacological management of hypertension in pregnancy. Semin Perinatol. 2014;38 (8):487-95. doi:10.1053/j.semperi.2014.08.016.

17. Scantlebury DC, Schwartz GL, Acquah LA, White WM, Moser M, Garovic VD. The treatment of hypertension during pregnancy: when should blood pressure medications be started? Curr Cardiol Rep. 2013;15 (11):412. doi:10.1007/ s11886-013-0412-0.

18. El Guindy AA, Nabhan AF. A randomized trial of tight vs. less tight control of mild essential and gestational hypertension in pregnancy. J Perinat Med. 2008;36 (5):413418. doi:10.1515/jpm.2008.060.

19. Berg CJ, Callaghan WM, Henderson Z, Syverson C. Pregnancy-related mortality in the United States, 1998 to 2005. Obstet Gynecol. 2011;117 (5):1229-1230. doi:10.1097/ AOG.0b013e31821763f2.

20. Seely EW, Ecker J. Clinical practice. Chronic hypertension in pregnancy. N Engl J Med. 2011;365 (5):439-446. doi:10.1056/NEJMcp0804872.

21. Report of the National High Blood Pressure Education Program Working Group on High Blood Pressure in Pregnancy. Am J Obstet Gynecol. 2000;183 (1):S1-S22.

22. Bramham K, Parnell B, Nelson-Piercy C, Seed PT, Poston L, Chappell LC. Chronic hypertension and pregnancy outcomes: systematic review and meta-analysis. BMJ. 2014; 348:g2301. doi:10.1136/bmj.g2301.

23. Cockburn J, Moar VA, Ounsted M, Redman CW. Final report of study on hypertension during pregnancy: the effects of specific treatment on the growth and development of the children. Lancet. 1982;1 (8273):647-649.

24. Georges MR, Aristide YR, Germain MD, et al. Hypertension in pregnancy at the teaching hospital of Yalgado Ouédraogo, Burkina Faso. Journal of Hypertension: Open Access. 2015;4 (2):1-7. doi:10.4172/2167-1095.1000199.

25. Yanit KE, Snowden JM, Cheng YW, Caughey AB. The impact of chronic hypertension and pregestational diabetes on pregnancy outcomes. Am J Obstet Gynecol. 2012;207 (4):333.e1-e6. doi:10.1016/j.ajog.2012.06.066.

Copyright $\odot 2020$ The Author (s); This is an open-access article distributed under the terms of the Creative Commons Attribution License (http://creativecommons.org/licenses/by/4.0), which permits unrestricted use, distribution, and reproduction in any medium, provided the original work is properly cited. 